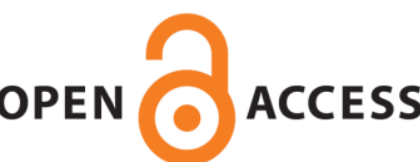

OPEN ACCESS

UWS Academic Portal

\title{
Resource efficient vehicle-to-grid (V2G) communication systems for electric vehicle enabled microgrids
}

Umoren, Ifiok A.; Shakir, Muhammad Z.; Tabassum, Hina

Published in:

IEEE Transactions on Intelligent Transportation Systems

DOI:

10.1109/TITS.2020.3023899

E-pub ahead of print: $23 / 09 / 2020$

Document Version

Peer reviewed version

Link to publication on the UWS Academic Portal

Citation for published version (APA):

Umoren, I. A., Shakir, M. Z., \& Tabassum, H. (2020). Resource efficient vehicle-to-grid (V2G) communication systems for electric vehicle enabled microgrids. IEEE Transactions on Intelligent Transportation Systems, 22(7), 4171-4180. https://doi.org/10.1109/TITS.2020.3023899

\section{General rights}

Copyright and moral rights for the publications made accessible in the UWS Academic Portal are retained by the authors and/or other copyright owners and it is a condition of accessing publications that users recognise and abide by the legal requirements associated with these rights.

Take down policy

If you believe that this document breaches copyright please contact pure@uws.ac.uk providing details, and we will remove access to the work immediately and investigate your claim. 
Umoren, I. A., Shakir, M. Z., \& Tabassum, H. (2020). Resource efficient vehicle-to-grid (V2G) communication systems for electric vehicle enabled microgrids. IEEE Transactions on Intelligent Transportation Systems, 1-10. https://doi.org/10.1109/TITS.2020.3023899

“ ( ) ( 2020 IEEE. Personal use of this material is permitted. Permission from IEEE must be obtained for all other uses, in any current or future media, including reprinting/republishing this material for advertising or promotional purposes, creating new collective works, for resale or redistribution to servers or lists, or reuse of any copyrighted component of this work in other works." 


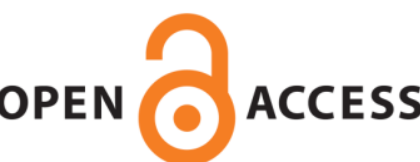

UWS Academic Portal

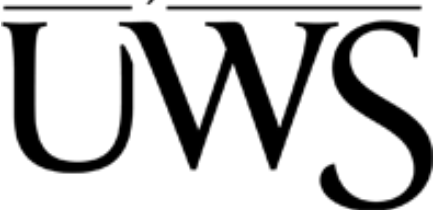

\section{Resource efficient vehicle-to-grid (V2G) communication systems for electric vehicle enabled microgrids}

Umoren, Ifiok Anthony; Shakir, Muhammad Zeeshan; Tabassum, Hina

Published in:

IEEE Transactions on Intelligent Transportation Systems

Accepted/In press: 08/09/2020

Link to publication on the UWS Academic Portal

Citation for published version (APA):

Umoren, I. A., Shakir, M. Z., \& Tabassum, H. (Accepted/In press). Resource efficient vehicle-to-grid (V2G) communication systems for electric vehicle enabled microgrids. IEEE Transactions on Intelligent Transportation Systems.

\section{General rights}

Copyright and moral rights for the publications made accessible in the UWS Academic Portal are retained by the authors and/or other copyright owners and it is a condition of accessing publications that users recognise and abide by the legal requirements associated with these rights.

Take down policy

If you believe that this document breaches copyright please contact pure@uws.ac.uk providing details, and we will remove access to the work immediately and investigate your claim. 


\title{
Resource Efficient Vehicle-to-Grid (V2G) Communication Systems for Electric Vehicle Enabled Microgrids
}

\author{
Ifiok A. Umoren, Muhammad Z. Shakir, Senior Member, IEEE, and Hina Tabassum, Senior Member, IEEE
}

\begin{abstract}
Intelligent vehicular communication is fundamental to manage vehicle-to-grid (V2G) interaction, where electric vehicles (EVs) provide energy to balance demand of critical loads (CLs). We propose resource efficiency (RE) to exploit the tradeoff between spectral efficiency (SE) and cost efficiency (CE) of EVs in a V2G communication network. The $\mathrm{CE}$ is the data rate of the V2G channel between EVs and base station (BS) over the operating cost of EVs to supply energy to CLs. We consider maximizing the RE in the downlink of a V2G communication network, where EVs are served by a BS and associated with CLs, while satisfying energy demand and charging station constraints. As the proposed RE problem is inherently non-convex and known to be NP-hard, we develop a suboptimal scheme based on a twophase algorithm. Phase 1 derives optimum EV-CL association using a heuristic approach, while phase 2 finds optimum power allocation using geometric programming. We then derive upper and lower bounds to the optimal RE as a benchmark to study the performance gap of the suboptimal scheme. Simulation results demonstrate that the proposed suboptimal scheme is close to the optimal solution, while its complexity is relatively low, making it promising for V2G applications.
\end{abstract}

Index Terms-Electric vehicle, vehicle-to-grid (V2G) communication, resource efficiency, convex optimization, microgrid, electric vehicle as a service (EVaaS).

\section{INTRODUCTION}

The growing popularity of electric vehicles (EVs) suggests that they could become key players towards unlocking future sustainable transportation and energy systems. EVs have numerous features, most notably the ability to be used as a service for intelligent transportation systems [1] and the electricity grid [2]. Vehicle-to-grid (V2G) technology enables EVs to interact with the electricity grid, where energy stored in the EV battery is supplied back to the grid to fulfill additional peak demand and mitigate the severity of power outages [3]. The traditional way of transporting the stored energy (from EV battery to consumer) through the grid leads to network losses and increased energy costs [4]. Network losses and energy costs can be reduced if EVs store, transport and supply the energy directly to the critical loads (CLs) [5]. This system requires a tightly controlled spectrum for reliable communication between EVs and the microgrid through the base station (BS) [6]. The system would need information about EVs

I. A. Umoren and M. Z. Shakir are with the School of Computing, Engineering and Physical Sciences, University of the West of Scotland, Paisley, Scotland, UK, Email: \{ifiok.umoren, muhammad.shakir\}@uws.ac.uk.

H. Tabassum is with the Department of Electrical Engineering and Computer Science, York University, Toronto, ON M3J 1P3, Canada, Email: hina.tabassum@1assonde.yorku.ca. such as battery capacity, energy price and location information to intelligently manage the energy resources distributed over large geographical areas. Transmitting this information over efficient wireless communication links is a crucial requirement for successful $\mathrm{V} 2 \mathrm{G}$ interaction.

\section{A. Background}

The standard protocols for communication over long distances in vehicular networks are IEEE 802.11p and WiMAX technologies [7]. The IEEE 802.11p standard and mobile WiMAX (based on IEEE 802.16e standard) have been investigated as a media for vehicular communication [8], [9]. IEEE $802.11 \mathrm{p}$ technology is the popular standard for vehicular networks, offering a coverage area of up to $1 \mathrm{~km}$, data rates of up to $54 \mathrm{Mbps}$ and latency as low as $50 \mathrm{~ms}$. On the other hand, WiMAX technology possess similar features as IEEE 802.11 p but offers longer range communication of up to $5 \mathrm{~km}$, higher data transfer speed of up to $100 \mathrm{Mbps}$ and very low delays between $25-40 \mathrm{~ms}$, which is suitable for V2G applications. The mobility mode of WiMAX standard is based on orthogonal frequency-division multiple access (OFDMA) that provides flexibility in radio resource management (RRM) and robustness against adverse effects of multipath fading. With OFDMA, exclusive channel assignment eliminates intracell interference, in which subchannels are allotted to a maximum of one EV in each cell at any given time [10].

RRM plays a major role in the performance of vehicular communication networks [11], [12]. The nature of the vehicular communication links based on the radio channel and the access to the shared resources causes variable available bandwidth, latency and data rate. This could prevent the correct operation of $\mathrm{V} 2 \mathrm{G}$ applications. The V2G network needs to be fast and efficient in order to support the increasing number of EVs expected to participate in V2G. Spectral efficiency (SE) is one of the key performance metrics in the design and optimization of wireless communication networks. It is an essential performance metric for evaluating the effectiveness of V2G networks. Cost efficiency (CE) is another performance indicator which measures the data rate of the V2G channel between EVs and BS over the operating cost of EVs expected to supply energy to CLs. Optimizing the SE does not actualise a cost-efficient V2G system, likewise, costeffective optimization does not achieve a spectrum-efficient V2G communication link. Hence, taking cost efficiency into consideration while achieving spectrum efficiency is both challenging and desirable. 
A number of recent works have focused on the use of wireless communication between EVs and the microgrid for V2G management [13]-[20]. An EV based V2G integrating virtual plant architecture is outlined in [13]. The study is focused on the $\mathrm{V} 2 \mathrm{G}$ communication requirement to gather data from EVs, the electricity grid, energy generators, and other grid subsystems as well as to communicate with EVs for control purposes. In [14], a framework for enabling IP communications in mobile $\mathrm{V} 2 \mathrm{G}$ environments is introduced. The technologies, block components and protocols needed for IPV6 support in V2G communications were specified. A smart charging management system for EVs charging station is proposed in [15], which consists of a centralized management system and single-node charging controllers. The system obtains charging data and transmits control instructions to the BS via GPRS and ZigBee. An EV charging scheduling scheme is developed in [16], considering the impact of data communication unavailability on the scheduling performance of a charging station. An optimization problem is then formulated and solved for an optimal policy that minimizes the cost due to performance degradation and power consumption.

In [17], two IEEE 802.11p-based quality of service schemes that enable EVs and smart grid interaction are presented for EV charging coordination and control. Using access points (APs), informed decision can be taken on which $\mathrm{EV}$ receives highest priority to access the communication link based on EV battery level, and availability and electricity cost at different charging stations. In [18], the average delay time for a group of charging EVs covered by one AP is modeled, based on Markov chain representation for the wireless IEEE 802.11 MAC protocol. The model further considers the effect of a lossy wireless link between charging EVs and the AP and its impact on the delay. An EV charging management scheme utilizing vehicular communication between EVs and APs based on IEEE 1609 WAVE and IEC 61850 standards is proposed in [19]. Using data obtained from participating EVs and by implementing a smart scheduling scheme, EVs that critically need power supply are catered for. A softwaredefined networking-based control scheme is developed in [20] for vehicular communication networks. The scheme obtains optimal association of vehicles with APs and further allocates appropriate transmission rates for both LTE and Wi-Fi communication to maximize the overall system quality of experience. Most contributions to RRM for V2G communication networks focus on EV charging coordination; to the best of our knowledge, the use of $\mathrm{V} 2 \mathrm{G}$ communication for $\mathrm{EV}$ discharging management where EVs are associated with CL has not been studied in the literature in this context.

\section{B. Our Contributions}

This work is an extension of our work in [2], where the V2G communication network of the electric vehicle as a service (EVaaS) framework was not studied. In this paper, we present an intelligent vehicular communication system to support $\mathrm{EV}$ interaction with the electricity grid. We focus on the resource efficiency (RE) optimization which selects suitable EVs with efficient V2G communication links and operating costs for balancing short-term demand supply mismatch and mitigating the severity of prolonged outages in a microgrid. EV data including location information, battery capacity and discharge efficiency, as well as CL location information and energy demand is needed for the association, which takes energy balance and charging station constraints into account. Charging stations are utilized as sources for associated EVs to provide power to the CL. The major contributions of this paper are as follows.

- We propose RE to exploit the tradeoff between spectral efficiency (SE) and cost efficiency (CE) of EVs associated with CLs, with a weighting coefficient controlling the balance between SE and CE. The RE is then formulated as an optimization problem under CL energy demand and charging station constraints.

- The optimization problem is known to be NP-hard, posing difficulties in finding the optimal solution. Towards solving this optimization problem, we derive upper and lower bounds to the optimal RE. We then develop a less complex suboptimal scheme, based on a two-phase algorithm, to solve the proposed RE problem. For a fixed transmit power allocation, phase 1 finds the optimal EV$\mathrm{CL}$ association using a heuristic approach, and subsequently, with this obtained EV-CL association solution, phase 2 derives the optimum transmit power allocation solution using geometric programming (GP).

- Simulation results demonstrate that the strategy is effective in matching EV with CL on demand, thus achieving better utilization of EV battery capacity. Numerical results reveal that the proposed suboptimal scheme is close to the optimal solution, while its complexity is relatively low, making it applicable to $\mathrm{V} 2 \mathrm{G}$ wireless communication networks and particularly promising to EVaaS application.

The rest of the paper is organized as follows. In Section II, the system model is described, as well as the spectralefficient and cost-efficient only designs. We formulate the RE optimization problem in Section III. In Section IV, we derive an upper bound on the optimal solution to jointly solve the optimization problem. In Section V, a suboptimal scheme with low complexity is proposed, its algorithm explained and its computational complexity analysed. Simulation results and their analysis are provided in Section VI, followed by concluding remarks in Section VII.

\section{SYSTEM MODEL}

\section{A. System Description}

The EVaaS system comprises of electrical and communication networks, however the latter is the system under study. The EVaaS communication network, as shown in Fig. 11. consists of BS, EVs, CLs, microgrid central controller (MGCC) and smart meters. All EVs are connected with BS through dedicated V2G communication channels. We assume the wireless link between EVs and the BS is based on WiMAX standard. Meanwhile, each CL communicates with BS to gather the status information about EVs. Information regarding $\mathrm{EV}$ location or where it will be in the next time frame, the 


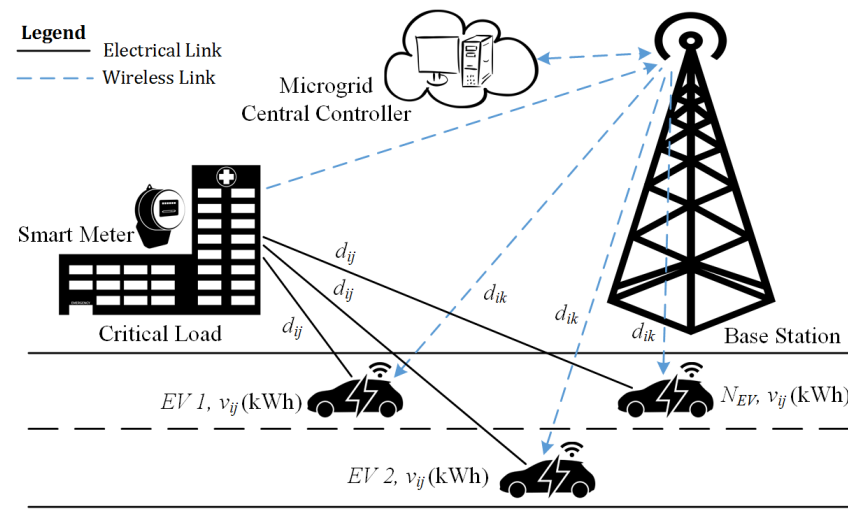

Fig. 1: EVaaS communication network consisting of a base station serving several electric vehicles.

amount of time and available energy to transport EV to CL, the amount of energy that will be reserved for trading and subsequently transporting $\mathrm{EV}$ back to its destination or a suitable charging station are essential for scheduling EV with the CL. The smart meter measures electricity consumption and responds to remote command operations, while the MGCC facilitates the automated coordinated operation between EVs and CLs. The MGCC also carries out various functions including demand response activities, economical scheduling of charging/discharging EVs and forecasting studies.

We consider the downlink of a V2G communication network, where the coverage of a specific area is provided by a single BS. The sets of EVs, CLs and BS are denoted as $i=\{1,2, \ldots, I\}, j=\{1,2, \ldots, J\}$ and $k=\{1,2, \ldots, K\}$, respectively. The total available bandwidth, $B_{t o t}$, is divided into $N$ subchannels, with the bandwidth of the $i$ th EV $b_{i}=$ $\left(B_{t o t} / N\right)$. With OFDMA, an exclusive channel assignment is assumed in each cell, i.e., subchannel cannot be assigned to more than one EV at any given time, to avoid interference among different EVs. It is to be noted that we investigate a scenario where the EVs are connected to a single serving base station at any given time and each BS optimizes its resources as if it was insulated from the others. Therefore, we have assumed no intercell interference from neighbouring BSs. We assume that the distribution of BS, EVs and CLs does not change for time duration $T$ (e.g., $T=10 \mathrm{~ms}$ ), and thus we study their association considering the active EVs during the time interval $[0 \mathrm{~T}]$.

The propagation channel between BS to EV is subject to Rayleigh fading, log-normal shadowing and a distant dependent path loss. We denote the channel gain between BS and $\mathrm{EV}$ as $H_{i}$, which is modelled as $H_{i}=\left|h_{i}\right|^{2} d_{i k}^{-\xi}$, where $\left|h_{i}\right|^{2}$ denotes the composite channel gain between BS and EV due to Rayleigh fading and log-normal shadowing, $\xi$ is the pathloss exponent, and $d_{i k}$ is the distance between the $k$ th $\mathrm{BS}$ and $i$ th EV.

We denote the transmit power allocated to the $i$ th EV over the V2G channel as $p_{i}$. Assuming full channel information is known at the $\mathrm{BS}$ and $\mathrm{EV}$, the received signal-to-noise ratio (SNR) at the $i$ th $\mathrm{EV}$ is expressed as:

$$
\gamma_{i}\left(p_{i}\right)=\left(\frac{p_{i} H_{i}}{N_{0} b_{i}}\right),
$$

where $N_{0}$ is the noise spectral density. Without loss of generality, noise spectral density is assumed to be equal for all EVs. It will be recalled that there is no interference among EVs due to assumption of OFDMA. The data rate of the $i$ th EV can be calculated by Shannon capacity formula as

$$
r_{i}\left(p_{i}\right)=b_{i} \log _{2}\left(1+\gamma_{i}\left(p_{i}\right)\right) .
$$

\section{B. Performance Metrics}

1) Spectral Efficiency: The SE refers to the data rate transmitted over the V2G channel between EVs and BS over the bandwidth of the EVs. The spectral efficiency of the $i$ th EV can be written as

$$
S E_{i}=\frac{r_{i}\left(p_{i}\right)}{b_{i}} .
$$

2) Cost Efficiency: The CE refers to the date rate of the V2G channel between EVs and BS over the operating cost of EVs expected to supply energy to CLs. The operating cost is the total amount to deploy EVs as distributed generators in a microgrid to supply CLs. The operating cost here includes the energy cost and the transportation cost to CL from the current location of EVs. The energy cost should cover the charge cost and compensate battery related losses incurred during during EV-CL interaction, to avoid making financial losses from EVaaS participation. Similarly, the transportation cost should cover transportation related liabilities. Factors used to calculate transportation fares include cost of vehicle parts, labour, road tax, insurance, fuel (energy) and average energy consumption rate $(0.2 \mathrm{kWh}$ per $\mathrm{km})[21]$. Considering these factors, we have assumed a transportation tariff for participating EVs. The operating cost is formulated as

$$
C_{i j}=E C_{i} v_{i j}+T C_{i} d_{i j},
$$

where $v_{i j}$ denotes the amount of energy of the $i$ th $\mathrm{EV}$, in $\mathrm{kWh}$, which is the on-demand battery capacity $v c_{i}$ multiplied by the discharge efficiency $e f_{i}$ of the battery $\left(v_{i j}=v c_{i} \cdot e f_{i}\right) ; E C_{i}$ denotes the unit energy cost of the $i$ th $\mathrm{EV}$, measured in British Pounds (£) per $\mathrm{kWh} ; T C_{i}$ denotes the unit transportation cost of the $i$ th EV, measured in British Pounds (£) per km; $d_{i j}$ denotes the estimated transportation distance between the $i$ th $\mathrm{EV}$ and $j$ th $\mathrm{CL}$, in $\mathrm{km}$.

The cost efficiency, measured in bits per second/British Pounds (£), can be written as

$$
C E_{i j}=\frac{r_{i}\left(p_{i}\right)}{C_{i j}} .
$$

\section{Resource EfFiciency Problem Formulation}

The RE exploits the tradeoff between spectral efficiency and cost efficiency. The objective of the optimization problem is to maximize the two objective functions, SE and CE, while satisfying all inequality and equality constraints. Considering the inconsistency of metric units for CE and SE, it is inappropriate 
to directly add CE and SE. Moreover, a simple summation of $\mathrm{CE}$ and $\mathrm{SE}$ will tend to focus the optimization problem on $\mathrm{CE}$, since the operating cost $C_{i j}$ is numerically smaller than the bandwidth $b_{i}$. Similar to [22], we now introduce a normalization constant $\beta^{\prime}=\beta\left(B_{t o t} / C_{t o t}\right)$, where $B_{t o t} / C_{t o t}$ acts as unit normalizer for SE and $\mathrm{CE}$, while the weight factor $\beta$ controls the balance of SE and CE.

Hence, the RE can be defined as

$$
R E_{i j}=\frac{r_{i}\left(p_{i}\right)}{C_{i j}}\left(1+\beta \frac{\eta_{C}}{\eta_{B}}\right),
$$

where $\eta_{C}$ and $\eta_{B}$ denotes operating cost utilization and bandwidth utilization, respectively, given by

$$
\eta_{C}=\frac{C_{i j}}{C_{t o t}}, \quad \eta_{B}=\frac{b_{i}}{B_{t o t}} .
$$

By substituting (7) in (6), we have

$$
R E_{i j}=C E_{i j}+\beta^{\prime} S E_{i} .
$$

Notably, due to normalization factor $B_{t o t} / C_{t o t}$, the unit of the proposed RE is bps/£, which is still the same as CE. On the other hand, the weight between $\mathrm{CE}$ and $\mathrm{SE}$ is $\beta$, such that the $\mathrm{RE}$ will optimize $\mathrm{CE}$ when $\beta=0$, while $\mathrm{SE}$ will be optimized when $\beta=\infty$. Choosing appropriate weight is then up to the decision maker, since there is no a priori correspondence between a solution vector and a weight vector [22]. Therefore, without loss of generality, $\beta$ is considered as a constant in our RE optimization problem.

Consider the system shown in Fig. 1, where EVs, CLs and BS are independently and identically distributed in a square region of area $A_{r}$. We model their locations as a binomial point process (BPP) [23]. This provides uniformly distributed random points of EVs, CLs and BS denoted as $\left(x_{i}, y_{i}\right),\left(x_{j}, y_{j}\right)$ and $\left(x_{k}, y_{k}\right)$, respectively. From the fixed positions of EVs, CLs and BS, the distance $d_{i j}=\mid x_{i}-$ $x_{j}|+| y_{i}-y_{j} \mid$ between $i$ th EV and $j$ th CL and distance $d_{i k}=\sqrt{\left(\left(x_{i}-x_{k}\right)^{2}+\left(y_{i}-y_{k}\right)^{2}\right)}$ between $i$ th EV and $k$ th $\mathrm{BS}$ is derived.

The association between EVs and CLs is constrained by some limiting factors, and would vary with changes in these factors. Hence, out of the available EV-CL pairs only few can be associated. The following discussion introduces the limiting factors of the system and then formulates the association problem of EVs and CLs based on those factors. $\alpha_{i j}$ is an entry of $(I \times J)$ association matrix $\alpha$ that defines the association of EVs and CLs as follows:

$$
\alpha_{i j}= \begin{cases}1, & \text { if } i \text { th EV is assigned to } j \text { th } \mathrm{CL} \\ 0, & \text { otherwise }\end{cases}
$$

The objective is to maximize the RE of EVs serving CL demand, provided they have available energy and are connected over efficient V2G communication links. The generalised RE optimization problem can be formulated as

$$
\max _{\alpha_{i j}, p_{i}} \sum_{i=1}^{I} \sum_{j=1}^{J} \alpha_{i j} \frac{r_{i}\left(p_{i}\right)}{C_{i j}}\left(1+\beta \frac{\eta_{C}}{\eta_{B}}\right)
$$

Subject to

$$
\begin{aligned}
& C 1: \sum_{i=1}^{I} \alpha_{i j} v_{i j} \geq V_{j}, \forall j \\
& C 2: \sum_{i=1}^{I} p_{i} \leq P_{\max } \\
& C 3: \sum_{i=1}^{I} \alpha_{i j} \leq C S_{j}, \forall j \\
& C 4: \sum_{i=j}^{J} \alpha_{i j} \leq 1, \forall i .
\end{aligned}
$$

The energy supplied by the EVs must satisfy CL demand and the system losses, however there is no loss of load being considered. This can be written as $C 1$, where the sum available energy from associated EVs $v_{i j}$ equals or exceeds the CL demand $V_{j}$. Since the total transmit power of the BS is nonnegative and limited, the sum transmit power to all associated EVs is constrained by $C 2$, where $P_{\max }$ denotes the maximum transmit power at the base station for downlink transmission. $C 3$ shows that $j$ th $\mathrm{CL}$ can maintain a maximum number of discharging EVs as per charging station limit $C S_{j}$. $C 4$ imposes that each EV can be associated to a maximum of one $\mathrm{CL}$.

\section{Optimal Resource Efficiency Schemes}

The optimization problem introduced in (10) is a combinatorial mixed-integer non-linear programming (MINLP) problem. It has a non-convex objective function and involves nonlinear constraints with binary and continuous variables, i.e. $\alpha_{i j}$ and $p_{i}$, respectively. That is to say, (10) is a general NP-hard optimization problem. Therefore, even for small setups, it is generally very hard to solve the original problem in reasonable time based on branch-and-bound or exhaustive search techniques. Thus, we propose a decomposition of the original MINLP problem into a mixed-integer linear programming (MILP) and a non-linear programming (NLP) problem. The MILP problem associates optimum EVs with CL, while the NLP problem derives the optimum transmit power allocation over the V2G communication network. Considering this approach, solutions can be obtained in desired computational times as discussed next.

\section{A. Optimal Resource Efficiency in High Signal to Noise Regime}

The optimal solution for (10) can be computed in the high SNR regime by an exhaustive search over all possible combination of the association, assuming perfect knowledge of channel gains at MGCC. For each possible association, optimum transmit powers can be computed by transforming (10) into a GP [24]. It should be noted that transmit power allocation problem is in itself a known non-convex problem for the general SNR regime [25]. But in a high SNR regime, it can be reformulated as a convex GP problem where the objective is to minimize a posynomial. For a fixed set of association 
variables and considering a high SNR regime, the objective function in (10) can be rewritten as follows

$$
\max _{p_{i}} \sum_{i=1}^{I} \sum_{j=1}^{J} \alpha_{i j} \log _{2}\left(\frac{p_{i} H_{i}}{N_{0} b_{i}+\psi}\right)\left(\frac{1}{C_{i j}}+\frac{\beta}{C_{i j}} \frac{\eta_{C}}{\eta_{B}}\right) .
$$

Maximizing the SNR is equivalent to minimizing the noiseto-signal ratio

$$
\min _{p_{i}} \sum_{i=1}^{I} \sum_{j=1}^{J} \alpha_{i j}^{\prime} \log _{2}\left(\frac{N_{0} b_{i}+\psi}{p_{i} H_{i}}\right)
$$

where $\alpha_{i j}^{\prime}=\alpha_{i j}\left(\frac{1}{C_{i j}}+\frac{\beta}{C_{i j}} \frac{\eta_{C}}{\eta_{B}}\right)$ and $\psi$ is a constant. Equivalently, (12) can be reformulated for high SNR regime as follows

$$
\begin{aligned}
& \min _{p_{i}} \log _{2} \prod_{i=1}^{I} \prod_{j=1}^{J}\left(\frac{N_{0} b_{i}+\psi}{p_{i} H_{i}}\right)^{\alpha_{i j}^{\prime}} \\
& \text { subject to: C2 }
\end{aligned}
$$

Note that the denominator in (13) is a monomial and the numerator is a posynomial. The ratio of a posynomial to a monomial is also a posynomial. The problem formulated in (13) is a GP problem in standard form that can be solved optimally through efficient interior point methods [26] after performing the logarithmic transformation of variables [25]. This will be used later on to derive optimum transmit power allocation. Due to extreme high computational complexity associated with exhaustive search-based EV-CL association phase, it is not recommended to compute the optimal solution. Additionally, the GP-based transmit power allocation is restricted by high SNR assumption and centralized timeconsuming computations. In respect to the aforementioned facts, developing bounds and suboptimal RE schemes with reasonable computational complexity is desirable.

\section{B. Upper Bound on the Optimal Resource Efficiency}

An alternative approach to make the problem (10) more tractable is to use linear programming relaxation. This relaxation technique has been frequently used in the context of subchannel assignment in multiuser OFDMA systems to convert mixed-integer programming problems into convex optimization problems [22], [27]-[30]. Unlike the previous definition where $\alpha_{i j} \in\{0,1\}$ indicates whether the $i$ th EV is associated to the $j$ th CL, we need to relax the binary constraint on the association matrix $\alpha_{i j}$ as

$$
0 \leq \alpha_{i j} \leq 1
$$

so the entries of the association matrix are continuous and can vary between 0 and 1 . The fractional $\alpha_{i j}$ can be interpreted as time domain sharing of charging stations at the CL. The sharing factor converts (10) into a convex optimization problem, as illustrated in the following:

$$
\max _{\alpha_{i j}, p_{i}} \sum_{i=1}^{I} \sum_{j=1}^{J} \alpha_{i j} \log _{2}\left(1+\frac{p_{i} H_{i}}{\alpha_{i j} N_{0} b_{i}}\right)\left(\frac{1}{C_{i j}}+\frac{\beta}{C_{i j}} \frac{\eta_{C}}{\eta_{B}}\right)
$$

subject to: $\mathrm{C} 1-\mathrm{C} 4$.

Evidently, by relaxing $\alpha_{i j}$ as (14) and reformulating the RE objective function in (15), the RE optimization problem produces an upper bound on the RE of (10), although it does not always yield a solution where $\alpha_{i j}$ is either 0 or 1 . Recall that $C 4$ along with the binary condition (9) previously restricted each $\mathrm{EV}$ to be associated to a maximum of one CL only. This relaxation allows for the association problem to be solved using standard convex optimization techniques [31]. The complexity of the solution of (15) is comparably high to apply in practical V2G communication systems. More importantly, the solution of (15) is infeasible because the sharing factor $\alpha_{i j}$ (continuous between 0 and 1) means that an EV can be associated to multiple CLs, which is not the case of our considered problem, according to $C 4$. However, we can take the solution as an upper bound of the optimization problem (10) to benchmark the performance of the other schemes.

\section{Suboptimal Resource EfFiciency Schemes}

Convex programming is numerically stable but its computational complexity relies on the number of optimization variables, which can be large if the number of EVs and CLs are large. To tackle the computational complexity of the proposed optimization problem in (10), we split the procedure into two phases: EV-CL association phase and transmit power allocation phase. In phase 1, for a given transmit power allocation, the association matrix considered as the variable of the optimization problem is solved. This derived association matrix is then used in Phase 2 to find the corresponding allocated transmit power as the solution to the transmit power allocation optimization problem. Note that the EV-CL association phase involves an equal transmit power allocation step; thus, it is not totally independent of transmit power allocation.

\section{A. EV-CL Association}

Considering a fixed transmit power $p$, with uniform transmit power allocation scheme, the maximum achievable RE is defined as

$$
\max _{\alpha_{i j}} \sum_{i=1}^{I} \sum_{j=1}^{J} \alpha_{i j} \frac{r_{i}\left(p_{i}\right)}{C_{i j}}\left(1+\beta \frac{\eta_{C}}{\eta_{B}}\right)
$$

subject to: $\mathrm{C} 1-\mathrm{C} 4$.

In (16), the only optimization variable is $\alpha$, hence, (16) has less computational complexity compared to (10). When the optimal solution of (16) is found, EVs that offer the highest RE are assigned to the CLs. We can take the solution of (16) as the lower bound to measure the performance of other algorithms. 


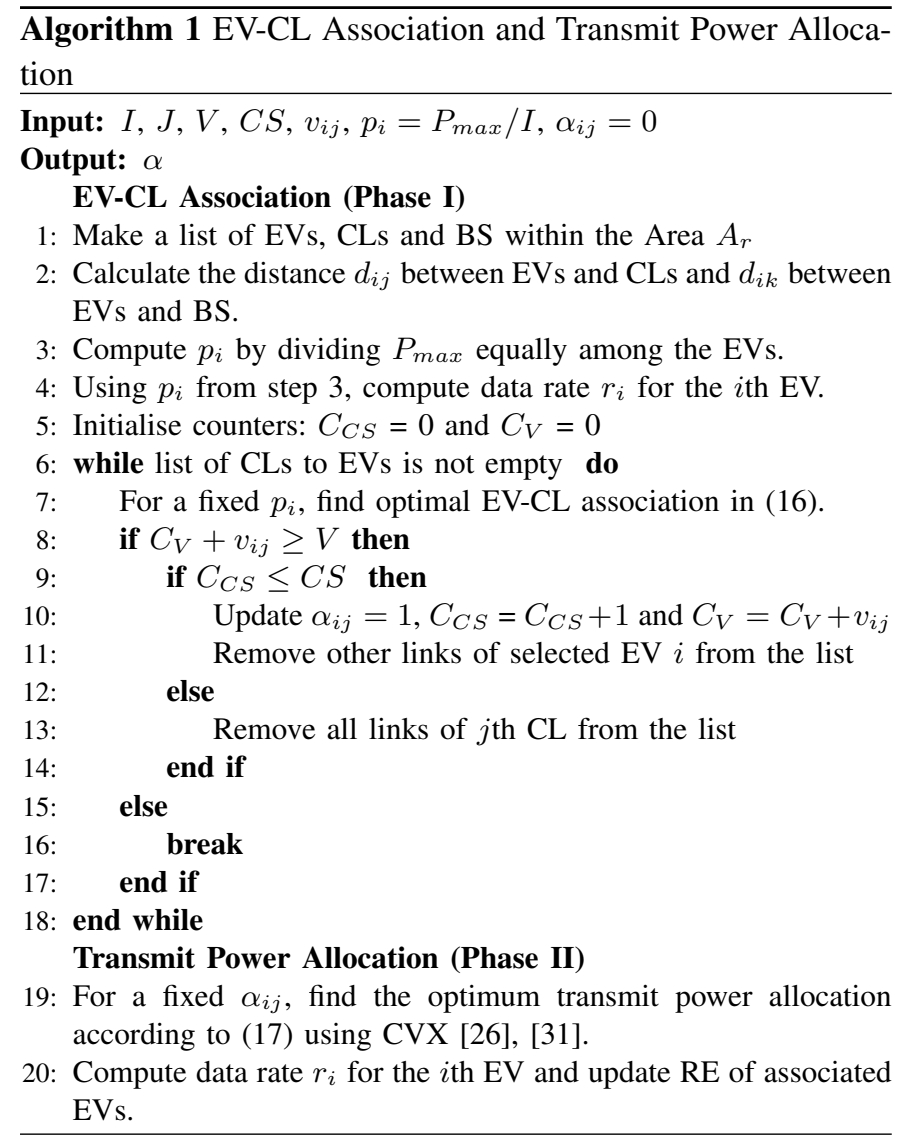

\section{B. Transmit Power Allocation}

Considering a fixed association matrix $\alpha$, the maximum achievable RE at a certain maximum transmit power, $P_{\max }$, is as follows

$$
\max _{p_{i}} \sum_{i=1}^{I} \sum_{j=1}^{J} \alpha_{i j} \frac{r_{i}\left(p_{i}\right)}{C_{i j}}\left(1+\beta \frac{\eta_{C}}{\eta_{B}}\right)
$$

subject to: $\mathrm{C} 2$.

We solve the problem in (17) to find out the optimal transmit power through GP (13) using successive convex approximation. The target of the transmit power allocation is to ensure that associated EVs achieve better performance.

\section{Optimization Algorithm}

We propose an algorithm that finds the EV-CL association and transmit power allocation for each $\mathrm{EV}$ in a $\mathrm{V} 2 \mathrm{G}$ communication network. To implement the algorithm, a central processing unit is needed to collect all the network information and perform the optimization. The proposed strategy is to choose EVs with the maximum RE. The algorithm starts with computing $I \mathrm{EVs}, J$ CLs and $K$ BS and their distribution in a defined area. A snap shot of EVs, CLs and BS is then obtained providing their respective distribution points $\left(x_{i}, y_{i}\right),\left(x_{j}, y_{j}\right)$ and $\left(x_{k}, y_{k}\right)$, which is then used to derive the distance $d_{i j}$ between $i$ th EV and $j$ th CL and the distance $d_{i k}$ between $i$ th $\mathrm{EV}$ and $k$ th BS. The EV capacity $v_{i j}$ is also obtained, along with the CL demand $V_{j}$ and number of charging stations $C S_{j}$.

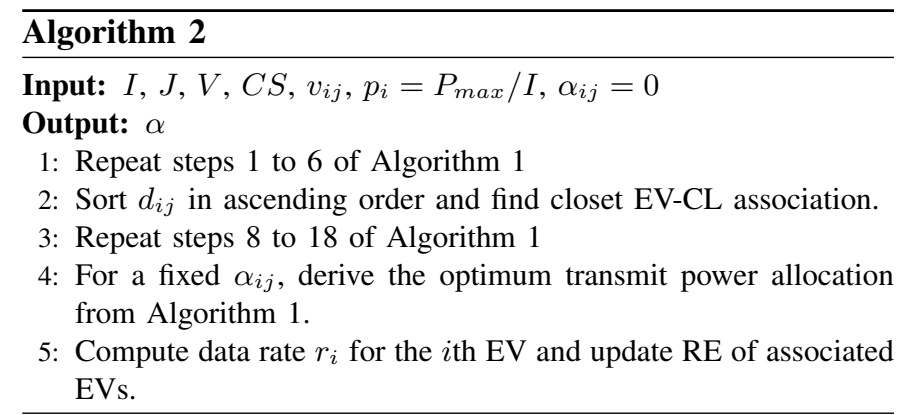

For the first phase, transmit power is divided equally over all EVs in the network. Two counters are initialized; energy demand of CLs $C_{V}$ and maximum number of charging stations $C_{C S}$. Out of the list of EV to CL links, the link that provides the optimal EV-CL association is selected. The algorithm then verifies the constraints of energy balance $C 1$ and number of charging stations $C 3$ such that $C_{V}+v_{i j} \geq V$ and $C_{C S}+1 \leq C S$, respectively. If all requirements are satisfied, the selected EV-CL pair is associated by modifying the association matrix entry as $\alpha=1$ and the respective counters are updated accordingly. The process repeats until the list ends or the resources ends that can be tracked using the two counters. Then, for a fixed association matrix $\alpha$, the optimal transmit power allocation (17) is found using GP (13). This is used to compute the data rate and update the RE of associated EVs. The steps are summarized in Algorithm 1.

We also consider a scenario where CL applies a predefined policy to select EVs with shortest geographic distance. This approach could be adopted during data communication unavailability from the MGCC. Since the decision making is always based on updated information, the system relies on the MGCC to develop effective optimal EV-CL association decision. However, issues related to transmission failure are usually inevitable. If optimum EV-CL association is not obtained from the system, the CL would select EVs with shortest distance as a backup scheme [32]. EVs are then associated with the CL based on first come first served (FCFS) scheme. In the FCFS scheme, the CL sorts the distances between EVs and CLs in an ascending order, and selects EVs that are closet to the CL. The strategy for the FCFS scheme is similar to Algorithm 1, with exception in the first phase. This is summarized in Algorithm 2.

\section{Computational Complexity}

Complexity plays a vital role in resource-efficient decisionmaking systems. Computations are performed in a centralised fashion, where a central controller has knowledge of the channel of all EVs and current association. Considering the size of problem (10) in our numerical analysis, it is not practical to find the optimal solution by any existing method. The global optimization method [33], which is applicable to smallscale problems, would take an impractical amount of time to return a global optimum of the transmit power allocation problem (17). We examine the computational complexity of our proposed algorithm considering float operations. At the first step, when EV is associated to a CL through linear search 


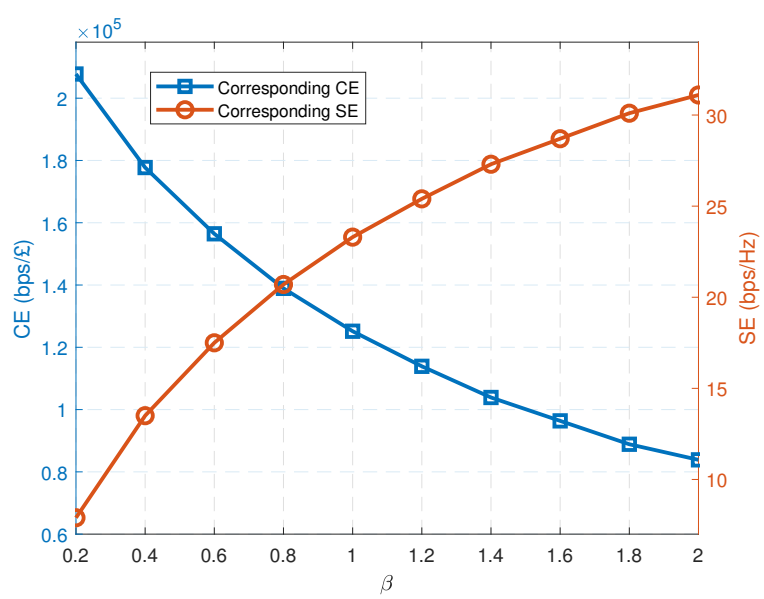

Fig. 2: Impact of weighted factor $\beta$ to the corresponding SE and CE.

in (16), the association matrix can be formed with a complexity of $O(I J)$. The while loop of the algorithm will terminate when the scheme defines the optimal $i$ th EV. When calculating the optimum transmit power allocation at the second step, the complexity is of $O\left(I \log _{2} I\right)$. Thus, the total complexity of our proposed algorithm is of $O\left(I J \log _{2} I\right)$.

Considering relevant literature, the computational complexity of the optimal solution (10) is significantly higher than that of our suboptimal scheme. Additionally, to the best of our knowledge, there is no other suboptimal solution available in previous related works that solves (10). Therefore, we limit our simulation study to demonstrating the performances of Algorithm 1 and Algorithm 2 and comparing the solutions in the upper bound and lower bound schemes.

\section{Simulation Results and Discussions}

We consider an EvaaS communication network where EVs, CLs and BS are BPP distributed within a $4 \mathrm{~km} \times 4 \mathrm{~km}$ area. We assume there are $I=12 \mathrm{EVs}$ and $J=2 \mathrm{CLs}$, unless otherwise stated. In the BS, we assume OFDMA downlink transmission with $K=32$ subchannels, each of which has a bandwidth of $15 \mathrm{kHz}$. The channel gains are derived by the Rayleigh fading model, where the path loss $\xi$ is set equal to 4 and $h_{i}$ is the exponential random variable with mean/parameter equal to 1 . The maximum transmit power $P_{\max }$ of $\mathrm{BS}$ is considered to be $1 \mathrm{~W}$, unless otherwise stated, and noise spectral density is chosen as $-174 \mathrm{dBm} / \mathrm{Hz}$. Battery capacity between $15 \mathrm{kWh}$ and $28 \mathrm{kWh}$ and discharging efficiency between 0.90 and 0.95 are randomly assigned to EVs. Unit energy tariff between $£ 0.07 / \mathrm{kWh}$ and $£ 0.12 / \mathrm{kWh}$ and unit transportation tariff between $£ 0.8 / \mathrm{km}$ and $£ 1.3 / \mathrm{km}$ are randomly assigned to EVs. Energy demand between 20 $\mathrm{kWh}$ and $200 \mathrm{kWh}$ is uniformly allocated to CLs. Considering the data of the EVs, CLs and BS, with battery capacity and coordinates for EVs, energy demand and coordinates for CLs, EvaaS communication network information and other parameters, the parameters for EV to CL association is computed. The necessary parameters are then passed to the algorithms
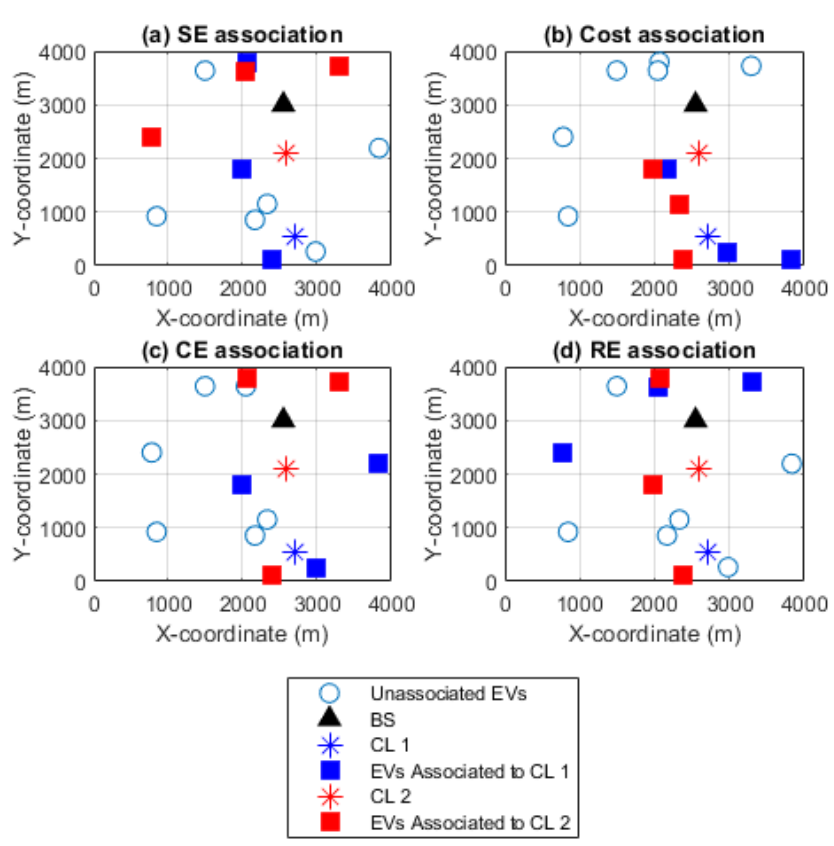

Fig. 3: The layout of random distribution and association of EVs and CLs with constraints $V=60 \mathrm{kWh}, C S=3$.

to find the best possible association between EVs and CLs by maximizing the optimization problem (10).

We present in Fig. 2 the impact of weighted factor $\beta$ to the corresponding SE and CE. Clearly, as the weight factor $\beta$ increases, the SE decreases, while the corresponding $\mathrm{CE}$ increases as the weight factor $\beta$ increases. Recall that RE focuses on optimizing $\mathrm{CE}$ when the value of $\beta$ is small, while the focus shifts to $\mathrm{SE}$ when the value $\beta$ is large. The reason is that an increase in $\beta$ results to putting more weight on SE, thus more resources (data rate and bandwidth) are allocated for SE maximization. Specifically, when $\beta=0.8$, the corresponding $\mathrm{CE}$ and SE are close to the maximum $\mathrm{CE}$ (when $\beta=0$ ) and maximum SE (when $\beta=\infty$ ), with the amount of degradation being $2 \%$ and $3 \%$ on $\mathrm{CE}$ and SE, respectively. This can be seen as justification for the proposed use of $B_{t o t} / C_{t o t}$ as the normalization factor in the RE formulation. Consequently, we set $\beta$ to 0.8 for the remainder of the simulation sections.

We optimize the same problem in (10) using different objective functions and study the performance of the objective function versus binary variable. Fig. 3 presents a considered scenario of the the distribution and EV-CL association under the different objective functions. Figs. $3 \mathrm{a}, 3 \mathrm{~b}, 3 \mathrm{k}$ and $3 \mathrm{~d}$ show EVs associated with CLs based on SE, operating cost, CE and RE, respectively. Although the same number of EVs are associated with CLs in Fig. 3 , different EV-CL association are realized in all scenarios. This means the performance is different in each considered scenario, which we will analyze later in Figs. 7 and 8 . The case study verifies the capability of the formulations and proposed strategy to match suitable EVs with CLs, thus achieving better utilization of the energy stored in EVs.

We then evaluate and compare the performances of all 


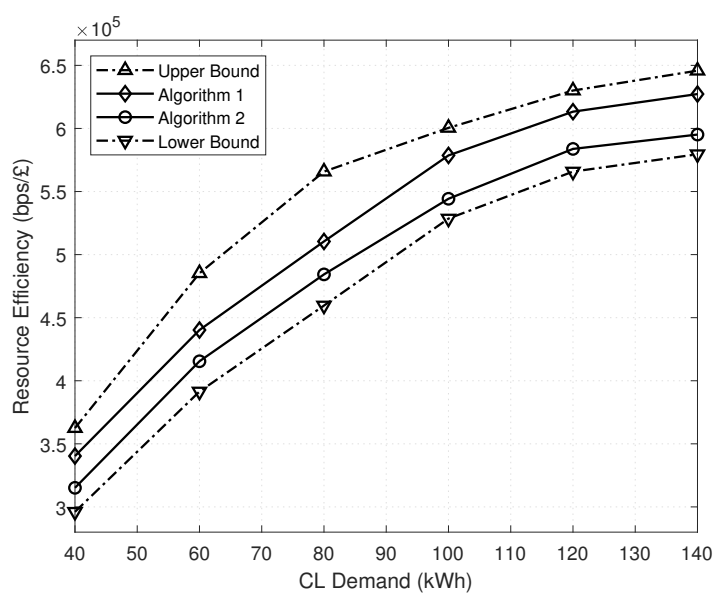

Fig. 4: Comparison of all proposed schemes for different CL demand.

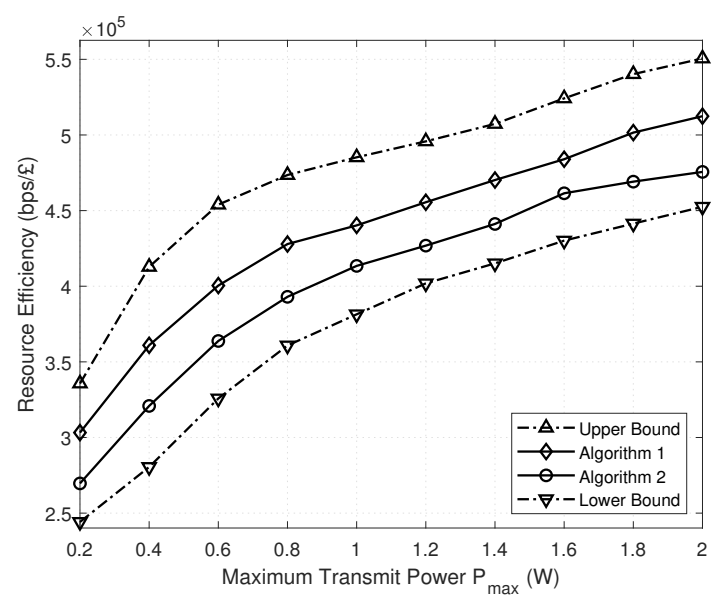

Fig. 5: Comparison of all proposed schemes for different maximum transmit power.

the proposed schemes. Fig. 4 shows the comparison of the maximum achievable RE obtained by Algorithm 1, Algorithm 2, upper bound (15) and lower bound (16) for different CL demand from $20 \mathrm{kWh}$ to $140 \mathrm{kWh}$. The first point to notice from Fig. 4 is that the maximum achievable RE is increased with an increase in the CL demand. This can be attributed to the increasing number of associated EVs. It can also be noticed that Algorithm 1 considerably outperforms Algorithm 2 for different CL demand. It can be interpreted as Algorithm 1 achieves better capacity utilization of EVs compared to Algorithm 2. In the absence of appropriate power allocation, the overall system performance will be impacted. The significance of the transmit power allocation phase can be observed easily from the performance gap between the Algorithm 1 and the lower bound. Algorithm 1 is close to the upper bound at certain CL demand. Specifically, Algorithm 1 results in a RE that is at least $91 \%$ of the upper bound RE. Although the upper bound is not idea for our case study and reflects an over-optimistic RE, it provides an idea on the performance gap between the proposed schemes and the

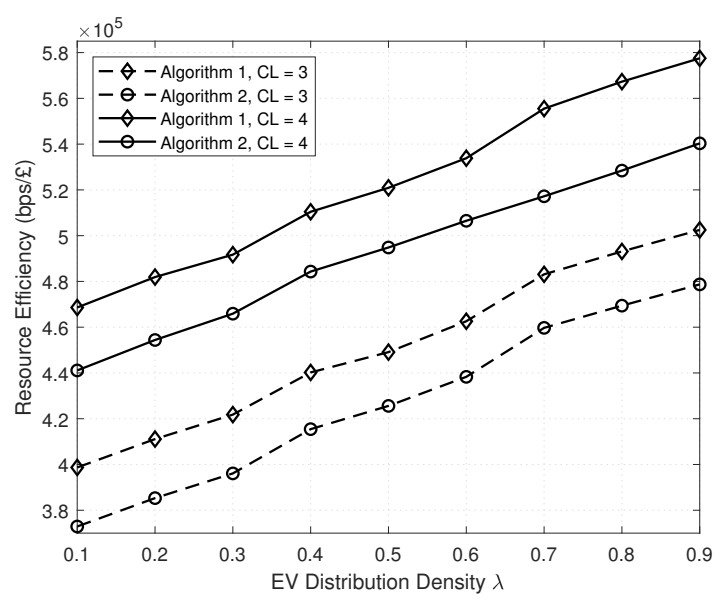

Fig. 6: Resource efficiency of associated EVs versus EV distribution density.

optimal solution.

We investigate the impact of different maximum transmit power $P_{\max }$ to the maximum achievable RE. Fig. 5 shows the comparison of the maximum achievable RE obtained by Algorithm 1, Algorithm 2, and the upper and lower bounds for different maximum transmit power from $0.2 \mathrm{~W}$ to $2 \mathrm{~W}$. As expected, with increasing maximum transmit power, the total maximum achievable RE also increases. However, there is a notably rise in the maximum achievable RE up to $1 \mathrm{~W}$ and then it slowly increases between $1.2 \mathrm{~W}$ and $2 \mathrm{~W}$. This shows that much power can be saved by lowering the maximum transmit power, since increasing it does not necessarily guarantee the best maximum achievable RE to transmission power budget ratio.

We present in Fig. 6 the impact of EV distribution density $\lambda$ to the maximum achievable RE. Here, the number of EVs is a random variable. For a single value of density, we have generated 100 different scenarios and then averaged the maximum achievable RE obtained by Algorithm 1. The low density represents rural areas with less EVs, while the high density represents their urban counterparts with much more EVs. As the density increases, the maximum achievable RE increases, as seen in Fig. 6. This demonstrates that the EVaaS system will achieve better RE in urban areas. Specifically, EVs will have better communication links and EVaaS applications will be cheaper, since more EVs are distributed closer to the CLs and BS. While the case study considers a microgrid with 2-4 CLs, the framework is general, and the extension is straight forward for multiple microgrids.

In Figs. 7 and 8, we compare the EV-CL association based on SE, cost, $\mathrm{CE}$ and RE functions. Fig. 7 shows the spectral efficiency for different CL demand. As expected, optimizing the SE function would typically give a better performances for spectral efficiency. However, the RE optimization produces a good trade off. The performance gap between the RE and CE functions is negligible, and they give nearly similar results, while the RE optimization gives a better performance than optimizing the cost function. Fig. 8 shows the operating cost for different CL demand. The cost optimization would 


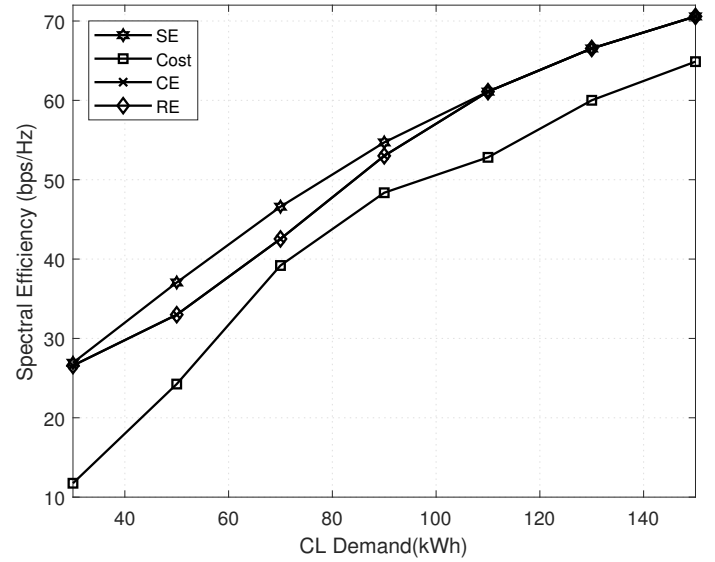

Fig. 7: Spectral efficiency of associated EVs for different CL demand.

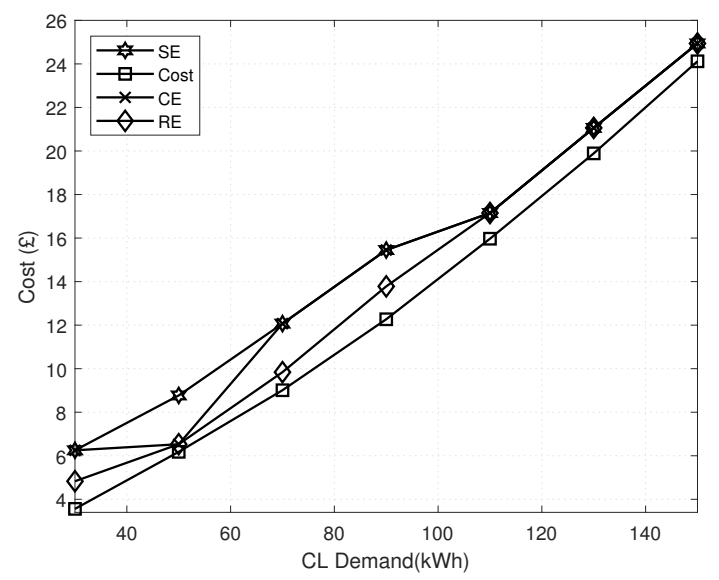

Fig. 8: Operating cost of associated EVs for different CL demand.

typically produces better results compared to the $\mathrm{SE}, \mathrm{CE}$ and RE functions. At CL demand of $70 \mathrm{kWh}$ and $90 \mathrm{kWh}$, the RE optimization produces a $23 \%$ and $12 \%$ improvement, respectively, compared to the $\mathrm{SE}$ and $\mathrm{CE}$ functions. While at $\mathrm{CL}$ demand of $30 \mathrm{kWh}$, the RE optimization produces a $30 \%$ improvement compared to the SE and CE functions. It is to be noted that the improvement by the RE optimization is not specific to low or high CL demand. The performance gap can be attributed to the different EV-CL association realized from optimizing the different objective functions, as seen in Fig. 3. Overall, optimization considering RE function gives the best performance. Thus, the proposed RE better utilizes the available resources in the EVaaS network.

\section{CONCLUSION}

In this paper, we present an intelligent vehicular communication system to enable $\mathrm{V} 2 \mathrm{G}$ interaction. We propose a resource efficiency framework for selecting suitable EVs to fulfil CL demand in V2G communication networks. The RE problem is formulated to maximize the spectral efficiency and cost efficiency, under CL energy demand and charging station constraints. In the proposed setup, EV-CL association and transmit power allocation are considered in a two-phase algorithm. Towards solving the proposed non-convex and NPhard optimization problem, we adopted an approach where in each phase, one set of optimization variables is derived. We also provide upper and lower bounds to the optimal RE as a benchmark to study the performance gap of the suboptimal scheme. Simulation results reveal that the performance of our proposed suboptimal scheme is close to the optimal solution, while the computational complexity is low, which makes it attractive for EVaaS applications. In future work, we will consider exploiting blockchain technology to establish a trusted environment for $\mathrm{V} 2 \mathrm{G}$ interaction [34]. EV interaction with the electricity grid is not secure and private; therefore, EVs are exposed to tracking, profiling and data breach using their mobile IP address. A blockchain-based system will preserve the data of participating EVs using different privacy protection techniques. Additionally, 5G networks will be utilized to address the communication requirements of the system.

\section{REFERENCES}

[1] G. Aujla, A. Jindal, and N. Kumar, "EVaaS: Electric vehicle-as-aservice for energy trading in SDN-enabled smart transportation system," Computer Networks, vol. 143, pp. 247-262, 2018.

[2] I. Umoren and M. Shakir, "EVaaS: A novel on-demand outage mitigation framework for electric vehicle enabled microgrids," in Proc. IEEE Globecom Workshops (GC Wkshps), Abu Dhabi, UAE, 9-13 Dec. 2018.

[3] W. Kempton and S. Letendre, "Electric vehicles as a new power source for electric utilities," Transportation Research Part D: Transport and Environment, vol. 2, no. 3, pp. 157-175, 1997.

[4] House of Commons Energy and Climate Change Committee, "Energy network costs: transparent and fair?" accessed on: May 20, 2019. [Online]. Available: https://publications.parliament.uk/pa/ $\mathrm{cm} 201415 / \mathrm{cmselect} / \mathrm{cmenergy/386/38602.html}$

[5] P. Yi, T. Zhu, B. Jiang, B. Wang, and D. Towsley, "An energy transmission and distribution network using electric vehicles," in Proc. IEEE International Conference on Communications (ICC), Ottawa, ON, Canada, 10-15 Jun. 2012.

[6] X. Hu, K. Wang, X. Liu, Y. Sun, P. Li, and S. Guo, "Energy management for EV charging in software-defined green vehicle-to-grid network," IEEE Commun. Mag., vol. 56, no. 5, pp. 156-163, May. 2018.

[7] D. Hoang, P. Wang, D. Niyato, and E. Hossain, "Charging and discharging of plug-in electric vehicles (PEVs) in vehicle-to-grid (V2G) systems: a cyber insurance-based model," IEEE Access, vol. 5, pp. 732-754, Jan. 2017.

[8] I. Msadaa, P. Cataldi, and F. Filali, "A comparative study between $802.11 \mathrm{p}$ and mobile WiMAX-based V2I communication networks," in Proc. 4th International Conference on Next Generation Mobile Applications, Services and Technologies, Amman,Jordan, 27-29 Jul. 2010.

[9] C. Ribeiro, "Bringing the wireless access to the automobile: a comparison of Wi-Fi, WiMAX, MBWA, and 3G," in Proc. 21st Annual Computer Science Seminar, 2005.

[10] D. Ngo, S. Khakurel, and T. Le-Ngoc, "Joint subchannel assignment and power allocation for OFDMA femtocell networks," IEEE Trans. Wireless Commun., vol. 13, no. 1, pp. 342-355, Jan. 2014.

[11] M. Ashraf, C. Liu, M. Bennis, and W. Saad, "Towards low-latency and ultra-reliable vehicle-to-vehicle communication," in Proc. European Conference on Networks and Communications $(E u C N C)$, Oulu, Finland, 12-15 Jun. 2017.

[12] J. Mei, K. Zheng, L. Zhao, L. Lei, and X. Wang, "Joint radio resource allocation and control for vehicle platooning in LTE-V2V network," IEEE Trans. Veh. Technol., vol. 67, no. 12, pp. 12218-12230, Dec. 2018.

[13] B. Jansen, C. Binding, O. Sundstrom, and D. Gantenbein, "Architecture and communication of an electric vehicle virtual power plant," in Proc. IEEE International Conference on Smart Grid Communications, Gaithersburg, MD, USA, 4-6 Oct. 2010. 
[14] S. Cespedes and X. Shen, "A framework for ubiquitous IP communications in vehicle to grid networks," in Proc. IEEE Globecom Workshops (GC Wkshps), Houston, TX, USA, 5-9 Dec. 2011.

[15] Z. Yuan, H. Xuand, H. Han, and Y. Zhao, "Research of smart charging management system for electric vehicles based on wireless communication networks," in Proc. IEEE 6th International Conference on Information and Automation for Sustainability, Beijing, China, 27-29 Sept. 2012.

[16] Q. Dong, D. Niyato, P. Wang, and Z. Han, "An adaptive scheduling of PHEV charging: analysis under imperfect data communication," in Proc. IEEE International Conference on Smart Grid Communications (SmartGridComm), Vancouver, BC, Canada, 21-24 Oct. 2013.

[17] I. Al-Anbagi and H. Mouftah, "WAVE 4 V2G: Wireless access in vehicular environments for vehicle-to-grid applications," Vehicular Communications, vol. 3, pp. 31-42, 2016.

[18] A. Bilh, K. Naik, and R. El-Shatshat, "Evaluating electric vehicles response time to regulation signals in smart grids," IEEE Trans. Ind. Informat., vol. 14, no. 3, pp. 1210-1219, Mar. 2018.

[19] S. Hussain, T. Ustun, P. Nsonga, and I. Ali, "IEEE 1609 WAVE and IEC 61850 standard communication based integrated EV charging management in smart grids," IEEE Trans. Veh. Technol., vol. 67, no. 8, pp. 7690-7697, Aug. 2018.

[20] W. Huang, L. Ding, D. Meng, J. Hwang, Y. Xu, and W. Zhang, "QoEbased resource allocation for heterogeneous multi-radio communication in software-defined vehicle networks," IEEE Access, vol. 6, pp. 33873399, Jan. 2018.

[21] "Huge differences in taxi fare costs," accessed on: May 8, 2020. [Online]. Available: https://www.sundaypost.com/news/uk-news/ huge-differences-in-taxi-fare-costs/

[22] J. Tang, D. So, E. Alsusa, and K. Hamdi, "Resource efficiency: a new paradigm on energy efficiency and spectral efficiency tradeoff," IEEE Trans. Wireless Commun., vol. 13, no. 8, pp. 4656-4669, Apr. 2014.

[23] D. Stoyan, W. Kendall, and J. Mecke, Stochastic Geometry and its Applications. John Wiley and Sons Ltd., 1995.

[24] H. Tabassum, Z. Dawy, and M. Alouini, "Resource allocation via sum-rate maximization in the uplink of multi-cell OFDMA networks," Wireless Commun. and Mobile Comput., vol. 11, no. 12, pp. 1528-1539, 2011.

[25] M. Chiang, C. Tan, D. Palomar, D. O'neill, and D. Julian, "Power control by geometric programming," IEEE Trans. Wireless Commun., vol. 6, no. 7, pp. 2640-2651, Jul. 2007.

[26] M. Grant and S. Boyd, CVX: MATLAB Software for Disciplined Convex Programming, 2007.

[27] C. Wong, R. Cheng, K. Lataief, and R. Murch, "Multiuser OFDM with adaptive subcarrier, bit, and power allocation," IEEE J. Sel. Areas Commun., vol. 17, no. 10, pp. 1747-1758, Oct. 1999.

[28] M. Tao, Y. Liang, and F. Zhang, "Resource allocation for delay differentiated traffic in multiuser OFDM systems," IEEE Trans. Wireless Commun., vol. 7, no. 6, pp. 2190-2201, Jun. 2008.

[29] W. Zhao and S. Wang, "Joint subchannel and power allocation in multiuser OFDM systems with minimal rate constraints," Intl. J. Commun. Syst., vol. 27, no. 1, pp. 1-12, 2012.

[30] C. Zarakovitis and Q. Ni, "Maximizing energy efficiency in multiuser multicarrier broadband wireless systems: convex relaxation and global optimization techniques," IEEE Trans. Veh. Technol., vol. 65, no. 7, pp. 5275-5286, Jul. 2016.

[31] S. Boyd and L. Vandenberghe, Convex Optimization. Cambridge, UK: Cambridge University Press, 2004.

[32] Y. Cao, N. Wang, G. Kamel, and Y. Kim, "An electric vehicle charging management scheme based on publish/subscribe communication framework," IEEE Syst. J., vol. 11, no. 3, pp. 1822-1835, Jul. 2017.

[33] L. Qian, Y. Zhang, and J. Huang, "MAPEL: Achieving global optimality for a non-convex wireless power control problem," IEEE Trans. Wireless Commun., vol. 8, no. 3, pp. 1553-1563, Mar. 2009.

[34] I. Umoren, S. Jaffary, M. Shakir, K. Katzis, and H. Ahmadi, "Blockchain-based energy trading in electric vehicle enabled microgrids," IEEE Consum. Electron. Mag., 2020, to be published, doi: 10.1109/MCE.2020.2988904.

\section{About the Authors}

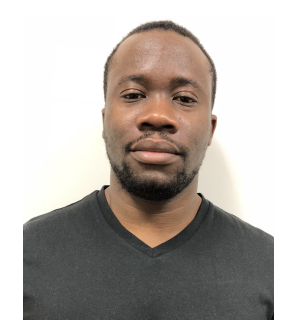

Ifiok Anthony Umoren is a $\mathrm{PhD}$ Student in the School of Computing, Engineering and Physical Sciences at University of the West of Scotland, UK. He received the MSc degree in Electronic and Electrical Engineering from Edinburgh Napier University, UK, and the BEng degree in Electrical and Electronics Engineering from the University of Port Harcourt, Nigeria in 2016 and 2013, respectively. His research interests include on-demand electric vehicle services, vehicular communication systems, energy trading, resource allocation and blockchain.

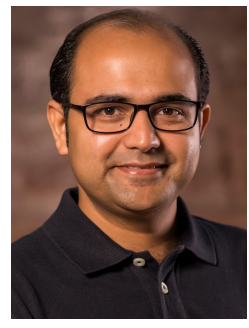

Muhammad Zeeshan Shakir is a Reader at the University of the West of Scotland, UK, received close to $£ 1 \mathrm{~m}$ funding from bodies such as Innovate UK, ERASMUS and matched by UK industries. With over 10 years of research expertise in design and development of wireless communication systems, he has published over 120 research articles and edited or contributed to 10 books. He is a recipient of STARS (Staff Appreciation and Recognition Scheme) Award 2018 for Outstanding Research and Enterprise performance at UWS. He is serving as a Chair of several symposiums/workshops in IEEE flagship conferences, including Globecom, ICC and VTC. He is an Editor of PHYCOM, IEEE Communications, and IEEE Communications Letters. He is a founding Chair of IEEE ComSoc emerging technical committee on backhaul/fronthaul. He is a Senior Member of IEEE and an active member of IEEE ComSoc.

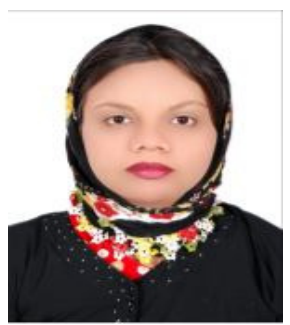

Hina Tabassum (SM'17) is currently an Assistant Professor at the Lassonde School of Engineering, York University, Canada. Prior to that, she was a research fellow at the Department of Electrical and Computer Engineering, University of Manitoba, Canada. She received the Ph.D. degree in communications engineering from the King Abdullah University of Science and Technology (KAUST), Saudi Arabia, in 2013. She was a recipient of two gold medals from the NED University of Engineering and Technology (NEDUET) and SIEMENS for securing the first position among all engineering universities of Karachi during her undergraduate studies and the Best Performance Award from Pakistan Space and Upper Atmosphere Research Commission (SUPARCO), in 2009. She is a Senior member of IEEE and registered Professional Engineer in the province of Ontario, Canada. She has been recognized as an Exemplary Reviewer (Top $2 \%$ of all reviewers) by IEEE Transactions on Communications in 2015, 2016, 2017, and 2019. Currently, she is serving as an Associate Editor in IEEE Communications Letters and IEEE Open Journal of Communications Society. Her research interests include 5G wireless networks with focus on their stochastic geometry-based performance modeling and optimization, internetof-things (IoT), and machine learning applications. 